$01 ; 03$

\title{
Численные исследования особенностей обтекания крыла в режиме бафтинга
}

\author{
(C) А.В. Воеводин, Д.А. Петров, А.С. Петров, В.Г. Судаков, Г.Г. Судаков \\ Центральный аэрогидродинамический институт им. проф. Н.Е. Жуковского, Жуковский, Московская обл., Россия \\ ฯE-mail: soudakov@mail.ru
}

Поступило в Редакцию 13 марта 2020 г.

В окончательной редакции 30 марта 2020г.

Принято к публикации 30 марта 2020г.

\begin{abstract}
Проведена валидация численного метода решения уравнений Рейнольдса для задачи обтекания прямоугольного крыла в режиме бафтинга и рассчитано обтекание прямоугольного крыла бесконечного удлинения безграничным околозвуковым потоком газа. Показано, что вместо двумерного течения реализуется течение, периодическое по боковой координате. Кроме того, имеют место периодические колебания течения по времени вдоль продольной координаты (бафтинг). Наличие боковых стенок трубы деформирует эту периодическую структуру и меняет частоту бафтинга. Расчет обтекания компоновки крыло-фюзеляж магистрального самолета в режиме трансзвукового бафтинга показывает, что колебания течения имеют место в ограниченной по размаху области крыла и происходят как в продольном, так и в поперечном направлении.
\end{abstract}

Ключевые слова: крыло, самолет, бафтинг, численный метод.

DOI: 10.21883/PJTF.2020.13.49583.18285

Бафтинг крыла при его околозвуковом обтекании представляет собой колебания потока, вызванные взаимодействием скачка уплотнения с отрывной зоной течения, возникающей за ним. При обтекании прямоугольного крыла большого удлинения безграничным потоком в этом режиме вместо плоского течения возникают периодические (по боковой координате) грибовидные структуры. Впервые они были обнаружены экспериментально [1] в дозвуковом отрывном режиме обтекания крыла, а затем воспроизведены численно [2]. Трансзвуковой бафтинг для случая обтекания профиля крыла исследовался экспериментально [3] и численно [4,5]. Режим бафтинга для модели самолета имеет значительно более сложный характер колебаний [6]. В [7,8] было показано, что начало бафтинга и появление грибовидных периодических структур связаны с глобальной неустойчивостью течения в отрывном режиме обтекания крыла. Механизм возникновения автоколебаний скачка уплотнения экспериментально исследован в [9], способ подавления в [10]. Главной темой указанных выше работ было исследование основных характеристик течения в режиме бафтинга: частоты и амплитуды пульсаций давления на поверхности крыла.

В настоящей работе исследовано обтекание прямоугольного крыла как безграничным потоком, так и при его установке в перфорированной рабочей части аэродинамической трубы (АДТ) с помощью численного метода для решения уравнений Рейнольдса при турбулентном состоянии пограничного слоя. Анализ результатов расчета позволил выявить некоторые дополнительные, ранее неизвестные особенности течения: для случая АДТ отрыв имеет место только в центральных и концевых сечениях крыла, средние (по размаху) сечения крыла обтекаются безотрывно, амплитуда колебаний скачка уплотнения зависит от боковой координаты. Кроме того, показано, что наличие стенок аэродинамической трубы сильно деформирует грибовидные структуры около боковых стенок и меняет частоту бафтинга на модели крыла (по сравнению со случаем обтекания крыла безграничным потоком). Показано также качественное отличие бафтинга для случая трансзвукового обтекания магистрального самолета от случая обтекания прямоугольного крыла.

Рассмотрим обтекание прямоугольного крыла, установленного в рабочей части АДТ Т-112 ЦАГИ. Размеры рабочей части составляют $-1.45<x<1.11 \mathrm{~m}$ в длину, $-0.32<y<0.32 \mathrm{~m}$ по вертикали, $-0.30<z<0.30 \mathrm{~m}$ по размаху. Модель прямоугольного крыла с хордой $b=0.2 \mathrm{~m}$ и размахом $L=0.6 \mathrm{~m}$ имеет поперечное сечение со сверхкритическим профилем с максимальной относительной толщиной 15\%. Торцы модели закреплены на боковых стенках рабочей части АДТ. Рабочая часть трубы имеет перфорированные верхнюю и нижнюю стенки. Коэффициент перфорации составлял $23 \%$.

В расчетных исследованиях в рамках уравнений Рейнольдса для учета перфорации стенок АДТ на нижней и верхней гранях ставятся условия Дарси

$$
v / U_{\infty}=R_{1,2}\left(p_{\text {plenum }}-p_{\text {wall }}\right) /\left(\rho_{\infty} U_{\infty}\right),
$$

где $v$ - вертикальная скорость на перфорированной грани, $\rho_{\infty}$ и $U_{\infty}-$ плотность и скорость набегающего потока, $p_{\text {plenum }}$ - давление в камере давления АДТ, $p_{\text {wall }}$ - давление на стенке, $R_{1,2}$ - коэффициенты проницаемости для вдува и отсоса через перфорированную грань соответственно. В наших расчетах в качестве $p_{\text {plenum }}$ использовалась величина статического давления из камеры давления АДТ: $p_{\text {plenum }}=p_{\infty}$. Предполагалось, 

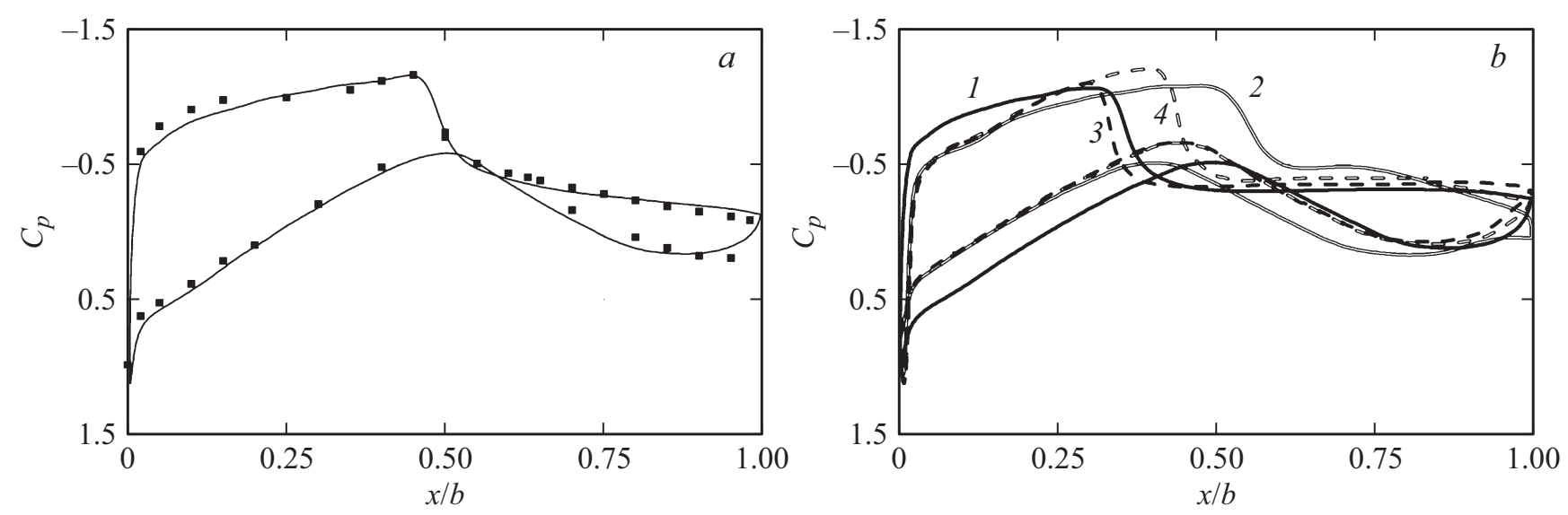

Рис. 1. $a$ - распределение коэффициента давления (среднее по времени) в центральном сечении крыла при $\mathrm{M}=0.77, \alpha=6^{\circ}$ в условиях АДТ: сплошная линия - расчет, точки - эксперимент. $b-$ распределение коэффициента давления (мгновенное, в фиксированный момент времени) при $\mathrm{M}=0.76, \alpha=6^{\circ}$ в сечениях крыла в условиях АДТ $(1,2)$ и в безграничном потоке $(3,4)$ : $z /(L / 2)=0(1), 0.47(2), 0.2(3)$ и $0.6(4)$.
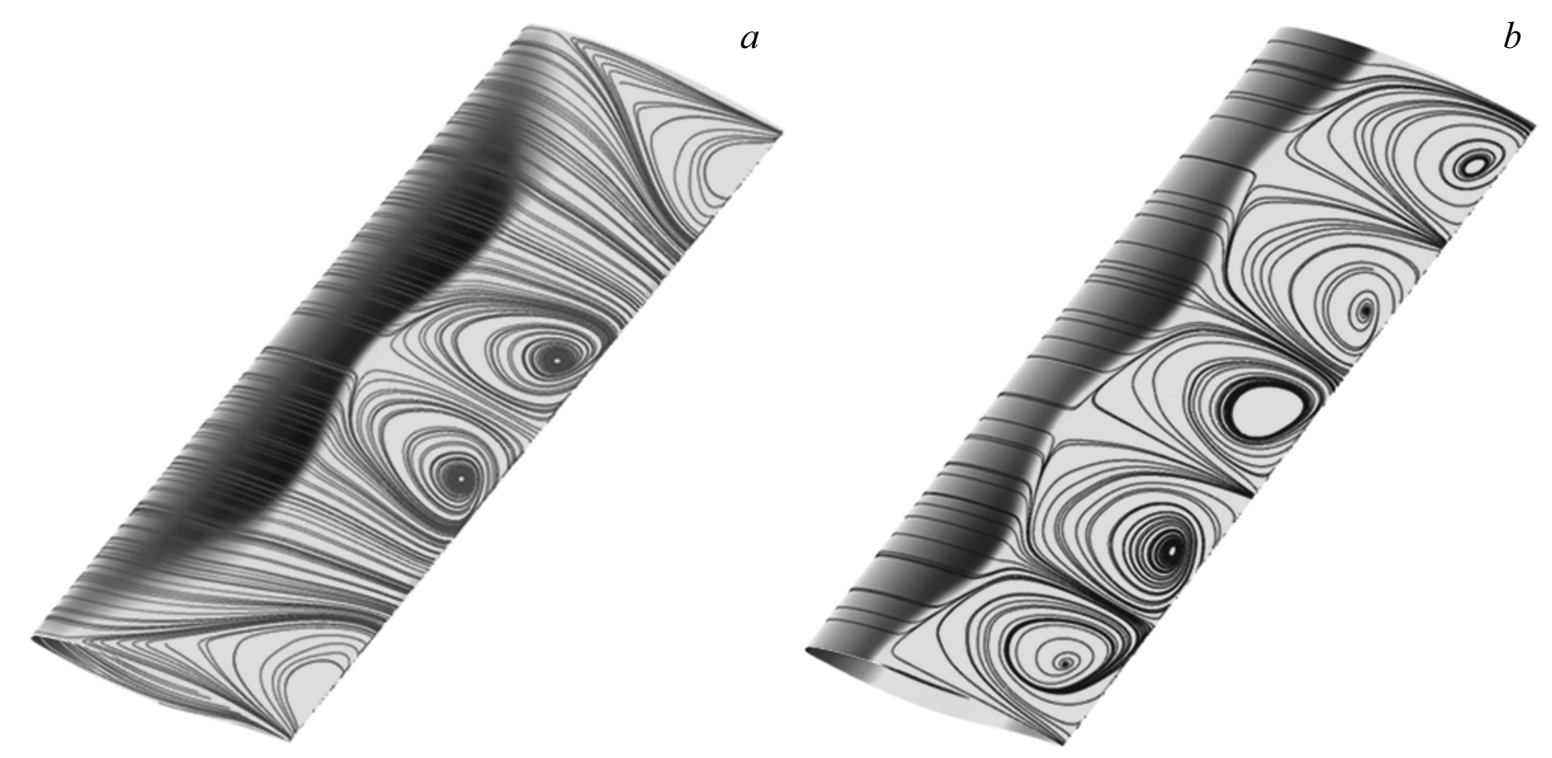

Рис. 2. Распределение давления и картины предельных линий тока на верхней поверхности модели крыла при $\mathrm{M}=0.76, \alpha=6^{\circ}$ в условиях АДТ $(a)$ и в безграничном потоке $(b)$ для крыла бесконечного удлинения в фиксированный момент времени.

что коэффициент проницаемости $R_{1}=R_{2}=R$. В случае сплошных стенок без перфорации коэффициент проницаемости $R=0$, а при наличии перфорации $-R=0.18$. Эта величина подбиралась из условия наилучшего совпадения расчетного и экспериментального [11] распределения давления на нижней поверхности рабочей части трубы как для безотрывного, так и для отрывного режима обтекания модели крыла. Эксперимент проводился с использованием турбулизаторов для фиксации перехода ламинарного пограничного слоя в турбулентный.

На входной (левой) границе расчетной области ставились граничные условия на полное давление $p_{0 \infty}=103200 \mathrm{~Pa}$ и полную температуру $T_{0 \infty}=25^{\circ} \mathrm{C}$. Профиль пограничного слоя на входе в рабочую часть АДТ не задавался. На выходной (правой) границе задава- лось статическое давление $p_{\infty}=70340 \mathrm{~Pa}$. На боковых стенках расчетной области ставились условия прилипания. Исследуемые числа Маха $\mathrm{M}=0.73-0.78$, число Рейнольдса слабо меняется с числом Маха и составляет около $\operatorname{Re}=3 \cdot 10^{6}$. Режим бафтинга был обнаружен в диапазоне $\mathrm{M}=0.76-0.78, \alpha=4-6^{\circ}(\alpha-$ угол атаки $)$.

Центральной проблемой для задачи расчета бафтинга крыла является правильное описание автоколебаний скачка уплотнения, возникающего на верхней поверхности крыла при трансзвуковых скоростях полета. Для расчетов использовался численный метод конечных объемов для решения уравнений Рейнольдса второго порядка точности. Валидация и настройка решателя этого численного метода проводились для задачи описания бафтинга на модели крыла в условиях АДТ Т-112 


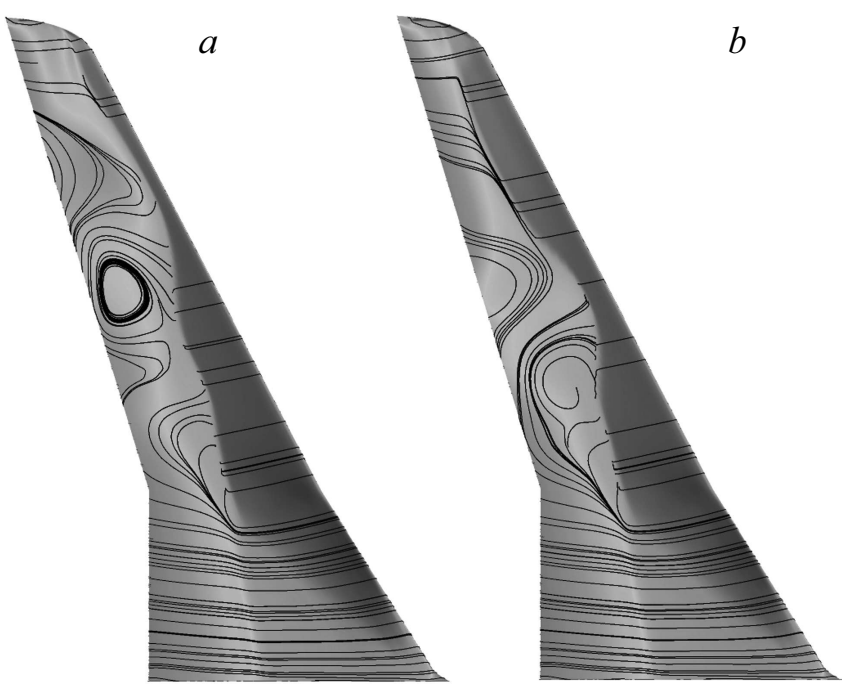

Рис. 3. Распределение давления и картины предельных линий тока на верхней поверхности крыла модели самолета при $\mathrm{M}=0.78, \alpha=5^{\circ} . a-$ начало периода, $b-$ половина периода.

ЦАГИ. Предварительные расчеты показали, что удовлетворительные результаты получаются при использовании однопараметрической модели турбулентности [12]. Для данной задачи к качеству сетки предъявляются повышенные требования, поэтому размер сетки для половины расчетной области составлял $2.4 \cdot 10^{7}$ узлов (использовалась структурированная расчетная сетка). Для случая крыла бесконечного удлинения в безграничном потоке расчеты проводились для всей расчетной области при использовании условий симметрии на боковых гранях вместо условия прилипания. На отодвинутой верхней и нижней гранях расчетной области ставились условия, соответствующие набегающему потоку. Размер расчетной сетки для всей расчетной области составлял $4.8 \cdot 10^{7}$ узлов.

Валидация численного метода проводилась как для безотрывного, так и для отрывного режима обтекания модели крыла. Среднее (по времени) распределение коэффициента давления $C_{p}$ на поверхности крыла в центральном сечении для условий АДТ при $\mathrm{M}=0.77$, $\alpha=6^{\circ}$ при отрывном режиме обтекания показано на pис. $1, a$. Наличие отрыва подтверждается возникновением отрицательных значений коэффициента давления в окрестности задней кромки. Видно, что согласование расчетных и экспериментальных данных довольно хорошее, несмотря на отрывной характер течения.

По результатам проведенных в настоящей работе расчетов установлено, что картины течения как в условиях АДТ, так и в безграничном потоке трехмерные и существенно отличаются друг от друга (рис. $1, b$ ). В условиях АДТ отрывное обтекание имеет место в центральных сечениях крыла (при $z /(L / 2)=0)$, а в средних сечениях $($ при $z /(L / 2)=0.47)$ крыло обтекается безотрывно. Для случая обтекания крыла безграничным потоком отрыв потока (хотя и разной интенсивности) имеет место для всех сечений крыла.

Валидированный численный метод позволяет анализировать как качественные, так и количественные характеристики течения (картину распределения давления на поверхности крыла, положение скачка уплотнения, картину поверхностных линий тока и т.д.). Картины предельных линий тока на верхней поверхности крыла (рис. 2) подтверждают предыдущие выводы и указывают на наличие грибовидных структур. Расчеты показывают, что амплитуда колебаний скачка уплотнения неравномерна по размаху крыла: для случая АДТ она велика в центральном сечении крыла $(\Delta x / b=0.15)$ и меньше в средних сечениях $(\Delta x / b=0.05)$.

При переходе от обтекания модели крыла в АДТ (рис. 2,a) к обтеканию безграничным потоком крыла бесконечного удлинения (рис. 2, $b$ ) трехмерная периодическая структура течения сохраняется. Следует отметить, что размер грибовидной структуры практически одинаков для случаев АДТ и безграничного обтекания. Наличие боковой стенки АДТ приводит к стабилизации отрыва потока в ее окрестности и возникновению существенной деформации трехмерной структуры течения в этой области (рис. 2,a). Безразмерная частота бафтинга $\mathrm{Sh}=b f / u_{\infty}(f-$ частота колебаний $)$ для случая обтекания крыла в АДТ при $\mathrm{M}=0.76, \alpha=6^{\circ}$ оказалась равной величине $\mathrm{Sh}=0.07$ (расчет) и 0.08 (эксперимент). Для случая безграничного потока эта величина равна $\mathrm{Sh}=0.11$ (расчет).

Для модели компоновки крыло-фюзеляж магистрального самолета течения усложняются. Для демонстрации этих эффектов было рассчитано обтекание модели самолета безграничным потоком воздуха с параметрами $\mathrm{M}=0.78, \operatorname{Re}=10.7 \cdot 10^{6}$ (по длине средней аэродинамической хорды), удлинение крыла $\lambda=9.8$, стреловидность крыла по передней кромке $\chi=25^{\circ}$. В силу симметрии геометрии модели самолета расчеты проводились для половины расчетной области, где использовалась многоблочная структурированная сетка размером $3 \cdot 10^{7}$ ячеек. Численно решались уравнения Рейнольдса с однопараметрической моделью турбулентности [12]. На рис. 3 показаны картины предельных линий тока в разные моменты времени. Отрыв имеет место не на всей поверхности крыла, а в ограниченной по размаху области на внешней части консоли крыла. Поэтому амплитуда колебаний аэродинамических характеристик модели относительно невелика. В частности, при $\alpha=5^{\circ}$ для коэффициента подъемной силы она составила 5.7\% c безразмерной частотой $\mathrm{Sh}=0.14$. Перемещение отрывных зон и вихревых структур происходит не только вдоль хорды, но и (даже в большей степени) вдоль размаха крыла.

Таким образом, в работе показано, что при обтекании прямоугольного крыла бесконечного удлинения в режиме бафтинга реализуется периодическое по боковой координате течение при наличии колебаний по времени в продольном направлении. Наличие боковых стенок 
АДТ деформирует эту структуру и меняет частоту колебаний. При обтекании компоновки крыло-фюзеляж колебания течения имеют место как в продольном, так и в поперечном направлении.

\section{Финансирование работы}

Работа выполнена при финансовой поддержке Российского научного фонда (проект № 16-19-10407).

\section{Конфликт интересов}

Авторы заявляют, что у них нет конфликта интересов.

\section{Список литературы}

[1] Gregory N., Quincey V.G., O'Reilly C.L., Hall D.J. Progress report on observations of three-dimensional flow patterns obtained during stall development on aerofoils, and on the problem of measuring two-dimensional characteristics. Technical Report C.P. N 1146. Aeronautical Research Council, 1971.

[2] Manni L., Nishino T., Delafin P.-L. // Comput. Fluids. 2016. V. 140. P. 260-269.

[3] Jacquin L., Molton P., Deck S., Maury B., Soulevant D. // AIAA J. 2009. 47. N 9. P. 1985-1994.

[4] Deck S. // AIAA J. 2005. V. 43. N 7. P. 1556-1566.

[5] Абрамова К.А., Рызков А.А., Судаков В.Г., Хайруллин К.Г. // Изв. РАН. Механика жидкости и газа. 2017. № 2. C. 173-180. DOI: 10.7868/S0568528117020037

[6] Sartor F., Timme S. // AIAA J. 2017. V. 55. N 4. P. 1230 1240.

[7] Crouch J.D., Garbaruk A., Magidov D., Travin A. // J. Fluid Mech. 2009. V. 628. P. 357-369.

[8] Crouch J.D., Garbaruk A., Strelets M. AIAA Paper 20183229. DOI: $10.2514 / 6.2018-3229$

[9] Сидоренко А.А., Будовский А.Д., Поливанов П.А., Вишняков О.И. // Письма в ЖТФ. 2017. Т. 43. В. 12. С. 59-67. DOI: 10.21883/PJTF.2017.12.44709.16738

[10] Брутян М.А., Волков А.В., Потапчик А.В. // Письма в ЖТФ. 2019. Т. 45. B. 21. C. 19-21. DOI: 10.21883/PJTF.2019.21.48467.17881

[11] Petrov A.V., Potapchik A.V., Soudakov V.G. Investigation of flow control over the super critical airfoil by tangential jet blowing at transonic speeds // 30th Congress of the International Council of the Aeronautical Sciences (ICAS 2016). Doejeon, Korea, 2016. Paper 2016-0173.

[12] Menter F.R. // ASME J. Fluids Eng. 1997. V. 119. N 4. P. 876884. 